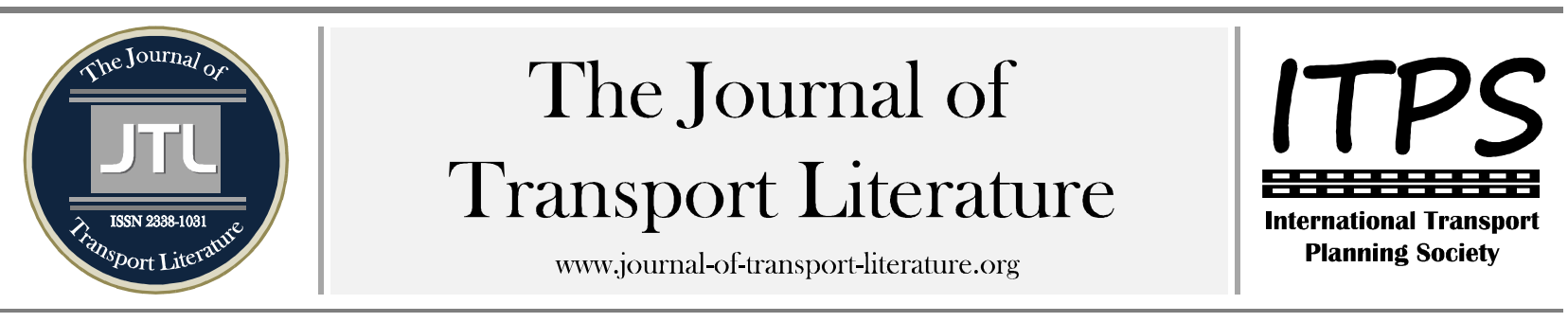

\title{
Aplicação do Método Paraconsistente de Decisão na Seleção de Tecnologias de Transporte Público Urbano
}

Sandro Gomes Rodrigues+; José Matsuo Shimoishi

Programa de Pós-Graduação em Transportes, Universidade de Brasília, Brasília-DF, Brasil

\section{Article Info}

Palavras-Chave:

método paraconsistente de decisão

baricentro

transporte público

Submitted 26 Jul 2014;

received in revised form 1 Dec 2014;

accepted 26 Dec 2014

Licensed under

Creative Commons

CC-BY 3.0 BR.

\section{Resumo}

Este trabalho tem como objetivo propor uma metodologia para seleção das diversas alternativas de transporte público, baseando-se na aplicação da lógica paconsistente em processos decisórios na área de planejamento de transportes. Utilizou-se o Método Paraconsistente de Decisão (MPD) como instrumento de apoio na seleção para o Eixo Sul do Distrito Federal (DF), no qual os especialistas da área de transportes avaliaram os critérios para cada modo, sendo a interpretação das avaliações realizadas na avaliação dos baricentros no quadrado unitário do plano cartesiano (QUPC). Com a aplicação do MPD na seleção de tecnologias de transporte verificou-se que todas as tecnologias de transporte pertencem a região de quase verdade tendendo ao paracompleto, tendo como análise não conclusiva. No entanto, na comparação das tecnologias, verifica-se a que possui maior grau de certeza é o Veículo Leve sobre Trilhos (VLT).

+ Corresponding author. SQN 113- Bloco D - Apto 208 - Asa Norte - Brasília /DF, Brasil.

E-mail address: sgomesrod@hotmail.com.

\section{Introdução}

O presente estudo tem como objetivo o propor uma metodologia para seleção das diversas alternativas de transporte público utilizando a lógica paraconsistente, buscando identificar dos fatores e atributos do processo, bem como a formulação do método paraconsistente de decisão aplicado na seleção de alternativas de transporte e sua aplicação. Para que ocorra essa seleção deve-se ter a preocupação em realizar uma análise criteriosa das características tecnológicas, dos passageiros a serem transportados, das condições urbanas e do sistema onde o modo de transporte será inserido. Nesse sentido, pode-se observar o nível de dificuldade na tomada de decisão, pois a seleção da tecnologia mais adequada está relacionada a fatores e atributos como custo, capacidade ofertada, segurança, potencial de atendimento em áreas centrais e periferias, impactos ao meio ambiente, infraestrutura disponível, etc.

No estudo da tomada de decisão vários métodos têm sido desenvolvidos. Entre as metodologias mais usadas no planejamento de sistemas de transportes, estão análises custo-benefício e abordagens multicritério. (INIESTRA e GUTIÉRREZ, 2009). Em apoio ao estudo de tomada de decisão, surgiu um novo método fundamentado em uma lógica alternativa à clássica, a lógica paraconsistente, que recebe o nome de Método Paraconsistente de Decisão (MPD), que consiste em um método que auxilia no processo decisório, utilizando a valoração dos especialistas de maneira a selecionar a alternativa adequada em função dos fatores estabelecidos, no qual possibilita verificar a indicação pela viabilidade (decisão favorável) ou pela inviabilidade (decisão desfavorável) de determinado projeto. (CARVALHO et al., 2003)

Para aplicação do MPD foram selecionados os fatores a partir de trabalhos da literatura de transportes de maneira a auxiliar nos critérios a serem avaliados. Os fatores e critérios foram utilizados no estudo de caso no Eixo Sul do DF, no qual os especialistas apresentaram suas avaliações quanto às tecnologias de transportes que poderiam ser implementadas, sendo verificada após a aplicação do MPD na que todas as tecnologias de transporte pertencem a região de quase verdade tendendo ao paracompleto, tendo como análise não conclusiva, sendo o grau de certeza do baricentro $\left(\mathrm{H}_{\mathrm{W}}\right)$, mantendo a mesma análise. No entanto na comparação das tecnologias, verifica-se a que possui maior grau de certeza é o VLT, necessitando maiores informações para que esta tecnologia seja a que melhor atendesse as necessidades para o Eixo Sul.

\section{Método Paraconsistente de Decisão (MPD)}

O Método Paraconsistente de Decisão (MPD) foi desenvolvido pelo estudo de Carvalho (2006), no qual buscou identificar fatores que influenciam no sucesso ou fracasso de um empreendimento, ou seja, que acabam influenciando na decisão de levar adiante ou não determinado projeto. Sua análise possibilitou identificar que os atributos podem em alguns casos indicar condições favoráveis, em outros desfavoráveis e em outros casos indiferentes. Esses fatores podem ser de diferentes ordens: econômicos, sociais, legais, ambientais, técnicos, políticos, entre outros. 0 método auxilia no processo decisório, utilizando a valoração dos especialistas de maneira a selecionar a alternativa adequada em função dos fatores estabelecidos, 
no qual possibilita verificar a indicação pela viabilidade (decisão favorável) ou pela inviabilidade (decisão desfavorável) de determinado projeto.

\section{Fatores e atributos utilizados para seleção de tecnologia de transporte público}

Ciarlini (2011) identificou os principais fatores e atributos que podem ser utilizados para seleção de tecnologias de transporte de massa, no qual levou em consideração a seleção de diversos trabalhos que abordavam a utilização de variáveis para a seleção de tecnologias de transportes.(Tabela 1)

Tabela 1 - Critérios para seleção de tecnologias de transporte.

Fonte: Adaptado de Ciarlini e Correia (2011).

\begin{tabular}{|c|c|c|c|c|}
\hline \multirow[t]{4}{*}{ CRITÉRIOS } & Aspectos econômicos & Aspectos da cidade & Aspectos dos usuários & Aspectos ambientais \\
\hline & Custo de projeto & Impactos no trífego & Cobertura do serviço & Interferências em áreas de veggetação urbana e parques \\
\hline & Custo da obra & Impactos em acidentes de trânsito & Tempos de viagem & Interferências em áreas de proteção ambiental \\
\hline & Custo de desapropriaçōes & Poluiģão visual & Número de transferências & Interferências em áreas de pastagens ou de agricultura \\
\hline \multirow[t]{4}{*}{ ATRIBUTOS } & Custo de reassentamento & Impacto em áreas históricas & Conectividade com outros modais & Interferências nos recursos hídricos \\
\hline & Custo operacional & Impactos no mercado imobiliário & & Qualidade do ar \\
\hline & & Impactos no uso do solo & & Ruidos \\
\hline & & Impactos na ocupação do solo & & \\
\hline
\end{tabular}

Corroborando com os estudos, Moraes (2012) apresenta a influência do fator político na tomada de decisão para projetos de transportes, apresentando os stakeholders que fazem parte do processo e o nível de influência na decisão. Observa-se que alguns aspectos são considerados, como a posição política, o relacionamento dos agentes envolvidos, as ações e recursos que possibilitam o convencimento e o interesse no projeto. Assim, pode-se considerar como fatores de influência em processos decisórios para seleção de tecnologias de transporte os aspectos econômicos, da cidade, dos usuários, ambientais e políticos, que interferem diretamente ou indiretamente em qualquer decisão relacionados a projetos de transportes.

\section{Metodologia proposta}

Para atender os objetivos deste trabalho, sinteticamente foram adotados sete macro-etapas fundamentais representadas na Figura 1:

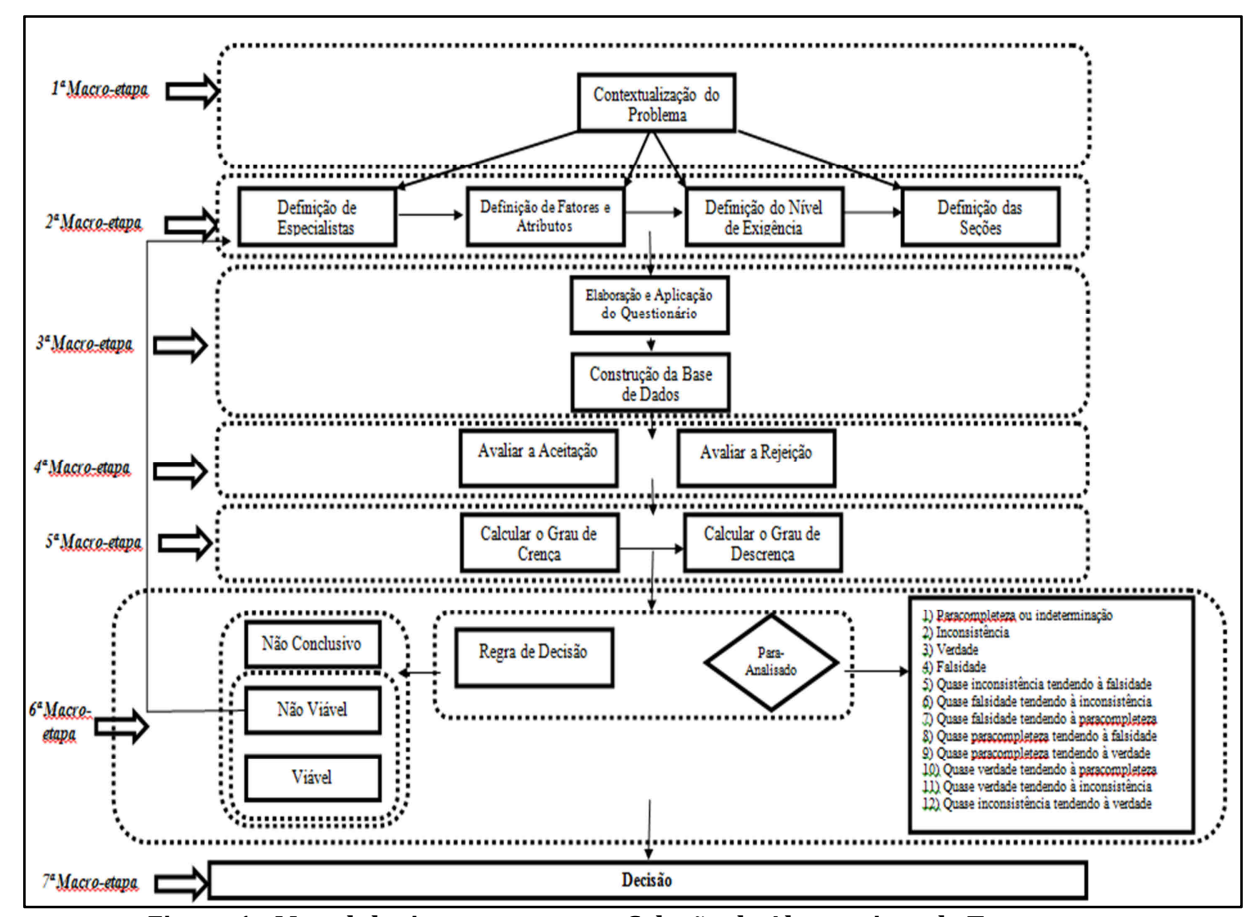

Figura 1 - Metodologia proposta para Seleção de Alternativas de Transporte.

Fonte: Rodrigues, 2013.

\section{Estudo de Caso}

Este estudo de caso compreenderá a seleção de alternativas de transporte público para o Eixo Sul do DF, utilizando a lista de critérios selecionada e pesos atribuídos pelos especialistas por meio do MPD. Serão analisadas as alternativas de transporte público urbano, baseada na metodologia proposta.

\subsection{Contextualização do Problema}

o projeto urbanístico de Brasília priorizou em sua concepção e ainda prioriza claramente a utilização do veículo individual no planejamento da cidade, o que resulta na forte vocação a este modo, principalmente automóveis e motocicletas, impactando negativamente no meio ambiente, colaborando para o aumento da poluição, e prejudicando a qualidade de vida da população com congestionamentos, acidentes e o aumento do tempo médio de deslocamento entre a casa e o trabalho. (CARVALHO, 2008)

Levando-se em consideração as principais vias que integram a infraestrutura viária do DF e seu Entorno, sobre as quais recai a pressão sobre o tráfego urbano, definem-se cinco eixos populacionais: Eixo Norte; Eixo Noroeste; Eixo Sul; Eixo Sudoeste; e Eixo Leste. Os eixos Noroeste e Sul reuniam, no ano 2000, mais de 56\% da população do DF e do Entorno. No 
Eixo Sul observa-se a diminuição da mobilidade devido ao fato das infraestruturas viárias existentes não atenderem as necessidades dos cidadãos, necessitando a construção de uma malha de vias rápidas ligando o sistema viário metropolitano e também a utilização de outras tecnologias de transporte. (CODEPLAN, 2012).

No processo de decisão para a seleção da alternativa de transporte para o Eixo Sul são consideradas as alternativas que foram levantadas junto aos especialistas por meio do questionário: Metrô; Veículo Leve sobre Trilhos (VLT); Ônibus Convencional; Bus Rapid Transit (BRT); Monotrilho; e Trem Urbano.

\subsection{Definição dos Especialistas}

Para a elaboração do modelo é necessária à seleção de especialistas que poderão apresentar seus conhecimentos, experiências, vivência, sensibilidade, intuição, bom senso, etc, para dar informações sobre as possibilidades da melhor tecnologia de transporte público para o Eixo Sul do DF, nas condições estabelecidas pelos atributos, para cada um dos fatores de influência escolhidos. A função do especialista é avaliar os atributos apresentados para cada tipo de transporte público descritos neste trabalho.. Os especialistas selecionados para participarem da pesquisa foram agrupados de acordo com sua experiência e conhecimento do produto adquirido: Grupo A (Ambiental); Grupo B (Consultor); Grupo C (Docente); Grupo D (Financeiro); e Grupo E (Gestor Público)

\subsection{Definição dos Critérios}

Nesta etapa devem-se definir os critérios que influem para seleção de alternativas de transporte para que se possa elaborar a base de dados com os fatores que influem no sucesso (ou fracasso) do projeto, bem como selecionar os mais importantes e de maior influência no processo decisório, ou seja, os que podem afetar acentuadamente a viabilidade ou não da tecnologia de transporte. Os critérios estabelecidos foram definidos pelo estudo desenvolvido por Ciarlini (2009) e Moraes (2012). Foram considerados os seguintes critérios: Econômicos; Cidade; Usuários; Ambientais; e Políticos.

\subsection{Definição do Nível de Exigência para o MPD}

Quanto ao nível de exigência aplicado nesse estudo, será estabelecido o valor de 0,50, pois no caso desse processo, a tecnologia selecionada será apenas para demonstrar a aplicação do método, sendo que a definição do tipo de transporte para o Eixo Sul já passou por uma avaliação. Assim, o nível de exigência é de maneira a diferenciar os tipos de transporte que podem ser implementados no Eixo Sul na opinião dos especialistas. Será considerado favorável ao tipo de transporte público urbano se, ao final, o grau de evidência favorável superar o de evidência contrária de 0,50, pelo menos. Com esse nível de exigência, o algoritmo para-analisador e a regra de decisão são apresentados na Figura 2. Para o nível de exigência de 0,5 consideram-se as seguintes avaliações: Viável quando o tipo de transporte esteja atendendo as exigências estabelecidas $(\mathrm{H} \geq 0,50)$; Inviável quando o tipo de transporte não esteja atendendo as exigências estabelecidas $(\mathrm{H} \leq-0,50)$; e Não Conclusivo quando é necessário novas informações para que se possa avaliar o tipo de transporte $(-0,50<\mathrm{H}<0,50)$.

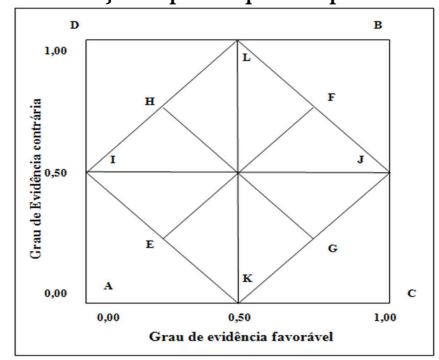

Figura 2 - Regra de decisão e algoritmo para-analisador para o NE igual a 0,50.

Fonte: Adaptado de Carvalho e Abe (2011).

\subsection{Definição dos atributos para o MPD}

Quanto aos atributos utilizados na seleção de tecnologias de transporte público para o Eixo Sul, serão considerados os critérios estabelecidos por Ciarlini (2009) e Moraes (2012), para os aspectos econômicos, da cidade, dos usuários, ambientais e políticos. (Tabela 1)

\subsection{Elaboração do questionário para seleção da tecnologia de Transporte Público}

A elaboração consistiu na proposta da colaboração dos especialistas de maneira a selecionar a tecnologia de transporte público para o Eixo Sul do DF. Assim, neste caso foram escolhidos " $n$ " fatores " $F_{i}$ " que influem na decisão, onde para cada fator são estabelecidos " $m$ " atributos " $\mathrm{S}_{\mathrm{j}}$ " que traduzem os atributos utilizados para seleção de transporte de massa.

\subsection{Aplicação dos questionários aos especialistas selecionados}

Foi apresentado aos especialistas selecionados, para que eles emitissem sua opinião do Grau de Aceitação e Rejeição de cada tipo de transporte a ser selecionado para o Eixo Sul. Como "especialista" na área, cada um deveria atribuir o valor "A" da evidência favorável (Aceitação ou Grau de crença) e o valor "R" da evidência contrária (Rejeição ou Grau de Descrença) na seleção da tecnologia de transporte público para o Eixo Sul. Ou seja, para cada fator " $F_{i}$ " nas condições determinadas pela seção Sj, estabeleceu-se dois valores das evidências, favorável (Aceitação) e contrária (Rejeição). Foi usada a opinião dos especialistas, baseada em seu background, ou seja, conhecimento, experiência, vivência, intuição, sensibilidade, bom senso etc. Os valores estabelecidos das evidências favorável (a) e contrária (b) são números reais que variam no intervalo fechado [0;1], ou seja, são números que podem variar de 0 (ou $0 \%$ ) a 1 (ou 100\%), incluindo estes. Esses valores não são complementares como acontece em probabilidades, isto é, não é necessário que $\mathrm{a}+\mathrm{b}=1$.

\subsection{Construção da base de dados}

Para construção da base de dados, foram utilizados os especialistas selecionados, com suas opiniões, por meio dos valores da evidência favorável (Graus de Crença) e da evidência contrária (Graus de Descrença) que cada um atribui para a escolha das tecnologias de transportes. A base de dados consistiu na divisão entre os grupos A, B, C, D e E, que representam 
a valoração apresentada em cada indicador para cada tipo de transporte. Os graus de crença e descrença de cada critério constituíram-se pelo valor médio das avaliações de cada especialista.

\subsection{Obtenção do Grau de Contradição e de Certeza para cada indicador com a utilização dos parâmetros lógicos} paraconsistentes

Com os valores do grau de crença (GÇ) e do grau de descrença (GDÇ) é possível estabelecer o grau de contradição (GCT) e o grau de certeza (GC). A Tabela 2 apresenta os resultados da aplicação do operador Max e Min para as tecnologias de transporte para o Eixo Sul para cada critério utilizado na análise. Foi efetuada a maximização dos Grupos A, B, C, D e E. Em seguida, realizou-se a minimização sobre os resultados obtidos e ao fim, aplicou-se o cálculo do grau de aceitação e rejeição para cada indicador. Foram calculadas as médias (W) para cada GC e GD, no qual se refere à solução para cada critério considerado (F1, F2, F3, F4 e F5).

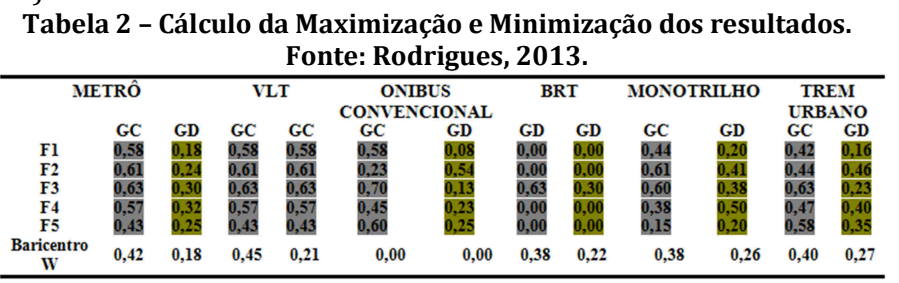

4.10. Avaliação final dos dados resultantes considerando a aplicação das regras de decisão e do algoritmo paraanalisador

Conforme a metodologia descrita inicialmente, foram calculadas as médias dos resultados dos GC e GD (Tabela 3). Estes resultados foram aplicados na regra de decisão e no algoritmo para-analisador ao nível de exigência de 0,50.

Tabela 3 - Aplicação da Regra de Decisão. Fonte: Rodrigues, 2013.

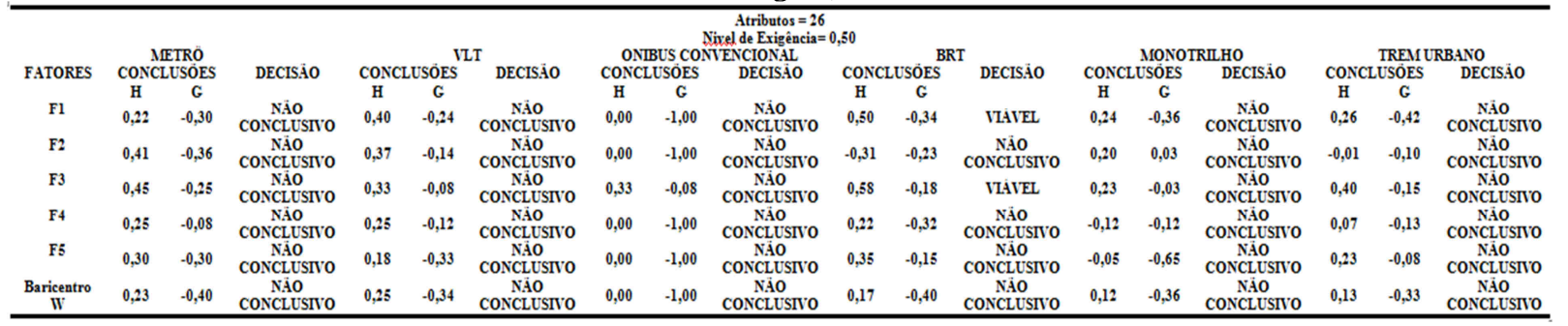

Outra forma da análise das tecnologias de transporte para o eixo é por meio da representação gráfica que apresenta o fluxo das informações utilizando a maximização e minimização dos resultados, obtendo ao final o GC e GD para cada tecnologias de transporte. A Tabela 4 apresenta os resultados obtidos para cada fator das tecnologias de transporte abordados no estudo.

Tabela 4 - Conclusões da análise de cada tecnologia de transporte.

\begin{tabular}{ccccc}
\multicolumn{5}{c}{ Fonte: Rodrigues, 2013. } \\
\hline Tecnologia de Transporte & $\mathrm{H}_{\mathrm{W}}$ & $\mathrm{NE}$ & Análise & Decisão \\
Metrô & 0,23 & 0,50 & $0,23<0,50$ & Não Conclusivo \\
VLT & 0,25 & 0,50 & $0,25<0,50$ & Não Conclusivo \\
Onibus Convencional & 0,00 & 0,50 & $0,00<0,50$ & Não Conclusivo \\
BRT & 0,17 & 0,50 & $0,17<0,50$ & Não Conclusivo \\
Monotrilho & 0,12 & 0,50 & $0,12<0,50$ & Não Conclusivo \\
Trem Urbano & 0,13 & 0,50 & $0,13<0,50$ & Não Conclusivo \\
\hline
\end{tabular}

\subsection{Tomada de Decisão}

Após a determinação dos valores de evidência favorável e contrária do baricentro, têm-se informações que possibilitam a auxiliar no processo decisório para definição da tecnologia de transporte para o Eixo Sul. Primeiramente plota-se o par ordenado no plano cartesiano de maneira a verificar a que região do reticulado o baricentro pertence (Tabela 4). A seguir verifica-se a localização do par-ordenado no baricentro para cada resultante das tecnologias de transporte. (Figura 3)

Pode-se verificar todos as tecnologias de transporte pertencem a região de quase verdade tendendo ao paracompleto, tendo como análise não conclusiva, ou seja, conclui-se nem pela viabilidade e nem pela inviabilidade de implantação, e caso seja de interesse deve-se realizar novos estudos de forma que se possa chegar a uma conclusão positiva (viabilidade ou inviabilidade), sempre tendo como apoio os estados não extremos correspondentes.

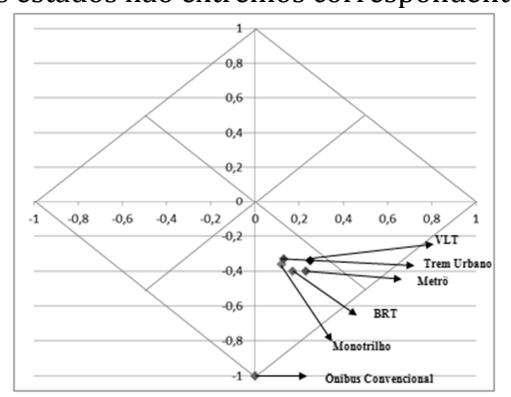

Figura 3 - Aplicação do algoritmo para-analisador para análise das tecnologias de transporte. Fonte: Rodrigues, 2013.

Outra análise para se definir a decisão final entre as tecnologias de transporte é pela aplicação da regra de decisão, no qual se calcula o grau de certeza do baricentro $\left(\mathrm{H}_{\mathrm{W}}=\mathrm{a}_{\mathrm{W}}-\mathrm{b}_{\mathrm{W}}\right)$ e aplicar a regra de decisão. 
a. Se $\mathrm{H}_{\mathrm{W}} \geq \mathrm{NE}$, a decisão é favorável e recomenda a execução do projeto (viável);

b. se $\mathrm{H}_{\mathrm{W}} \leq-\mathrm{NE}$, a decisão é desfavorável e recomenda a não execução do projeto (inviável); e

c. se $-\mathrm{NE}<\mathrm{H}_{\mathrm{W}}<\mathrm{NE}$, diz-se que a análise é não conclusiva.

A Tabela 4 apresenta o grau de certeza do baricentro $\left(\mathrm{H}_{\mathrm{W}}\right)$, no qual possibilita inferir que para todos as tecnologias de transporte tem-se como não conclusivo, corroborando com a análise feita pelo algoritmo para-analisador, que da análise o resultado não recomenda a implantação das tecnologias apresentadas e nem a não implantação, sugerindo apenas que, se for de interesse, novos estudos sejam feitos para se tentar resolver a indecisão.

No entanto, como o objeto de estudo era apenas realizar a comparação das tecnologias, busca-se como critério aquela que apresenta maior grau de certeza (Carvalho e Abe, 2011). Conclui-se que o VLT $\left(\mathrm{H}_{\mathrm{W}}=0,25\right)$ é a tecnologia selecionada nos parâmetros do baricentro, sendo que para implantação necessitaria maiores informações para que a tecnologia selecionada fosse a que melhor atendesse as necessidades para o Eixo Sul, considerando a opinião dos especialistas, seguidos do metrô $\left(H_{W}=0,23\right)$, BRT $\left(H_{W}=0,17\right)$, Trem Urbano $\left(H_{W}=0,13\right)$, Monotrilho $\left(H_{W}=0,12\right)$ e Ônibus Convencional $\left(H_{W}=0,00\right)$. (Tabela 4)

\section{Conclusão}

No desenvolvimento do estudo de caso pode-se constatar que apesar dos fatores e atributos utilizados permitirem uma comparação das alternativas de transporte percebeu-se a necessidade de um estudo mais detalhado dos atributos que possam ser utilizados na escolha da tecnologia de transporte. 0 estudo de caso possibilitou verificar a facilidade de aplicação do MPD, tanto na forma como é feita a avaliação pelos especialistas com a utilização da regra de decisão e do algoritmo para-analisador, quanto nas análises realizadas para se chegar aos resultados finais.

Considera-se que a metodologia proposta é de bastante utilidade como instrumento de auxílio à tomada de decisão sobre qual sistema implantar, considerando todos os aspectos relacionados com o transporte, ou seja, econômicos, cidade, usuários, ambientais e políticos. No entanto, necessita-se de maiores elementos que proporcionem aos especialistas condições de avaliarem para a decisão de implantação de determinada tecnologia, mostrando que o planejamento de transportes é algo complexo e que deve ser levantada todas as variáveis possíveis de maneira a minimizar os custos e os riscos da viabilização do projeto. 0 método pode ser utilizado como indicador para reformulação de novas pesquisas, e avaliar também o grau de coerência das respostas dadas.

Pode-se concluir que este método usando a Lógica Paraconsistente Anotada, oferece solução para processos de tomada de decisão no campo da administração. 0 caso apresentado associa-se à solução de problemas ligados à área de planejamento de transportes. Como exposto, a lógica paraconsistente fornece novas técnicas de análise mais apropriadas, principalmente na presença de dados incertos e contraditórios, permitindo manipular tais dados de modo não trivial, mostrando a necessidade de levantamento do maior número de informações possíveis para que a decisão tomada seja a mais acertada.

\section{Referências}

CARVALHO, D. L. (2008) Mobilidade urbana e cidadania no Distrito Federal : um estudo do programa Brasília integrada. Dissertação de Mestrado. Instituto de Ciências Sociais. Departamento de Sociologia da Universidade de Brasília. Disponível em: <repositorio.unb.br/bitstream/10482/1052/1/DISSERTACAO_2008_DiegoLourencoCarvalho.pdf> Acesso em: 20 Jun 2012.

CARVALHO, F. R.(2006) Aplicação de lógica paraconsistente anotada em tomadas de decisão na engenharia de produção. Tese de Doutorado. Escola Politécnica da Universidade de São Paulo. Disponível em: <www.teses.usp.br/teses/disponiveis/3/3136/tde-13032007-155453/pt-br. php> Acesso em: 15 Abr 2012.

CARVALHO, F. R., Carvalho, F. R., BRUNSTEIN, I e ABE, J. M. (2003) Um Estudo de Tomada de Decisão Baseado em Lógica Paraconsistente Anotada: Avaliação do Projeto de uma Fábrica. Revista Pesquisa e Desenvolvimento Engenharia de Produção n.1, pp. 47-62.

CIARLINI, M. e CORREIA, A. R. (2011) Aplicação de método multicritério no planejamento de sistema de transporte rápido de massa. Revista de Literatura dos Transportes, vol. 5, n. 3, pp. 192-209.

CODEPLAN (2012) Companhia de Planejamento do Distrito Federal. Disponível em: <www.codeplan.df.gov.br> Acesso em: 15 Mai 2012.

INIESTRA, J. G. e GUTIÉRREZ, J. G.(2009) Multicriteria decisions on interdependent infrastructure transportation projects using an evolutionary- based framework. Applied Soft Computing, vol 9, pp. 512-526.

MORAIS, A. C., (2012) Projetos de Infraestrutura de Transportes: Inserção efetiva na Agenda Governamental. Tese de Doutorado. Programa de Pós-Graduação em Transportes da Universidade de Brasília.

RODRIGUES, S. G. (2013) Aplicação da Lógica Paraconsistente na seleção de alternativas de transporte público. Dissertação de Mestrado - Universidade de Brasília. Faculdade de Tecnologia. Departamento de Engenharia Civil e Ambiental. Brasília. DF

\section{Abstract}

This paper aims to propose a methodology for selection of the various public transport alternatives, based on the application of paconsistente logic in decision-making processes in transport planning area. Was used the method of paraconsistent Decision (MPD) as a support tool in the selection for the South Axis of the Federal District (DF), in which experts from the transport area evaluated the criteria for each mode, and the interpretation of reviews at the evaluation of baricentres the Unit Square Cartesian Plane (QUPC). With the application of MPD in the selection of transport technologies it was found that all transmission technologies belong to region tending to paracompleto almost true, and as non-conclusive analysis. However, when comparing the technologies, there is the one with greater certainty is the Light Rail Transit (LRT).

Key words: paraconsistent decision method; barycentres; public transport. 